\title{
La familia Agavaceae en el eStado de Querétaro, México
}

\author{
fabiola Magallán Hernández y luis Hernández Sandoval \\ Escuela de Biología, Universidad Autónoma de Querétaro. \\ Centro Universitario, Querétaro 76010, Qro. México.e-mail: fabios@sunserver.uaq.mx
}

\begin{abstract}
Resumen. Se presenta un estudio floristico que incluye la identidad taxonómica y distribución de la familia Agavaceae en Querétaro. En México, las agaváceas son relevantes en la etnobotánica, la evolución, la florística y la taxonomía. Sin embargo, a pesar de que Querétaro posee gran diversidad ambiental, el conocimiento de la familia Agavaceae era incompleto, pues sólo se tenían 15 especies registradas en la literatura. Como resultado de este trabajo se encontraron seis géneros y 31 especies; dos de ellos (Beschorneria y Prochnyanthes) y 16 especies son nuevos registros. Se encontraron dos especies de Agave no descritas para la ciencia, y se conoce por primera vez la distribución natural de Agave albomarginata. Comparando el número de especies de Agavaceae por estados en México, por unidad de área, se concluye que Querétaro es el tercer estado más diverso.

Palabras clave: Agavaceae, florística, Querétaro, México.

Abstract. A floristic study including the taxonomic identity and distribution of Agavaceae was done for Querétaro, México. In Mexico, the family Agavaceae is very important for its etnobotanical, evolutionary, floristics and taxonomic relevance. Even that Querétaro has a high environmental diversity, the knowledge of the family was limited with previous reports of 15 species. By intensive collections, six genera and 31 species were found. Out of them, two genera (Beschorneria and Prochnyanthes) and 16 species are new reports for Querétaro. Besides, two species of Agave are new for science, and the natural distribution of Agave albomarginata is known for the first time. Comparing the number of species of Agavaceae per unit of area from each State in México, we conclude that Querétaro is the third more diverse state.
\end{abstract}

Key words. Agavaceae, floristics, distribution, Querétaro.

L a familia Agavaceae (Dahlgren et al., 1985) está formada por un grupo de plantas con o sin tallos aparentes, con hojas suculentas, coriáceas o herbáceas, en rosetas, flores bisexuales y semillas de color negro. Los ecosistemas áridos y semiáridos de México, en donde se presenta un alto grado de endemismo (Rzedowski, 1978), se distinguen por la presencia de muchas especies de agaváceas. Sin embargo, debe destacarse que esta familia ocurre en casi todos los ambientes, incluyendo áreas tropicales y templadas. Adicionalmente, las plantas de la familia Agavaceae tienen gran importancia en México desde los siguientes puntos de vista: $a$ ] florístico: ya que la familia es es endémica de América, con México representando el centro de mayor riqueza (García-Mendoza y Galván, 1995) con el $75 \%$ del total de especies y el $55 \%$ de endemismo (García-Mendoza, 1995); b] etnobotánico: muchas especies son útiles como alimento, bebida, vestido, utensilios y como ornamentales (Ojeda y Ludlow, 1995), formando parte de la cultura y religión de muchos pueblos indígenas (Gonçalves, 1978; Granados, 1993). De hecho, en numerosos lugares de Querétaro, la gente propaga especies como Agave americana, A. mapisaga y A. salmiana con fines de explotación y generalmente obtienen ingresos por la venta de pulque y aguamiel; $c$ ] taxonómico y evolutivo: en su historia, el grupo presenta una taxonomía confusa (han existido diferentes clasificaciones), debido a variación morfológica y la falta de estudios integrales que ayuden a entender las relaciones naturales del grupo y su evolución. Dado que México es el país con mayor diversidad de agaváceas, se han encontrado 
tanto especies con caracteres plesiomórficos como apomórficos, resaltando los problemas nomenclaturales (Hernández, 1998).

\section{Antecedentes florísticos y taxónómicos}

Los estudios florísticos de la familia Agavaceae en México han sido abordados tanto por los grupos taxonómicos que la conforman, como por las regiones o estados y a nivel nacional. En el aspecto taxonómico, uno de los primeros investigadores que estudió el género Agave en México fue Berger (1915), quien describe algunas especies, menciona su distribución e incluye un pequeño comentario para cada una. Trelease (1920), describe las especies mexicanas de Agave. Gentry (1982) publica uno de los estudios más completos hechos sobre el género Agave en América, con descripciones de las especies mexicanas.

Para Beschorneria, el único trabajo comprensivo de este género es el de García-Mendoza (1987) quien llevó a cabo una monografía en la cual se mencionan las especies distribuidas en México. Para el resto de géneros como Polianthes, Howard (1985) hizo un estudio en México, mencionando sus especies y distribución. Solano (en proceso) está haciendo una revisión moderna del mismo. Rose (1903-1905) estudió Manfreda, Polianthes y Prochnyanthes, presentando listas de especies para cada género. Verhoek-Williams (1975) llevó a cabo una revisión de los géneros Pro-

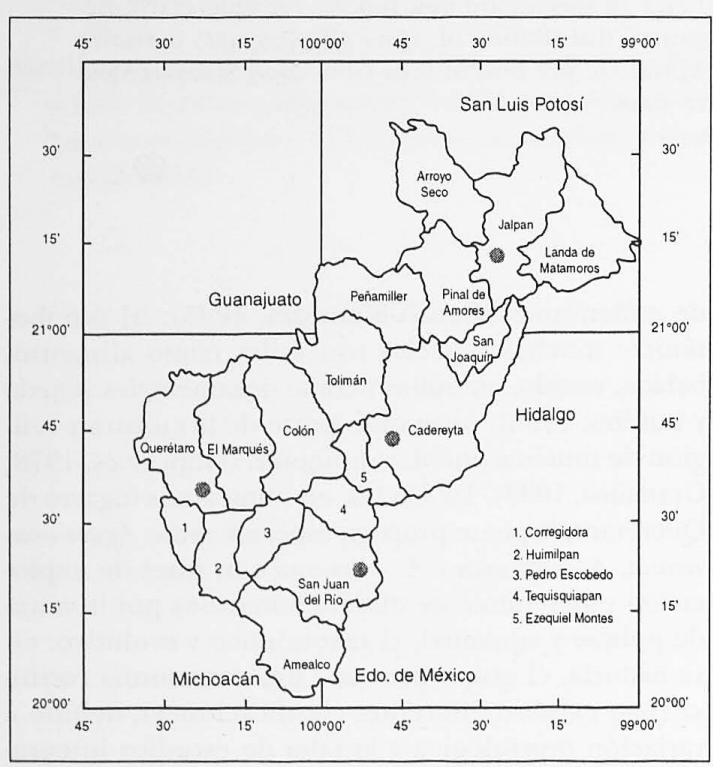

Figura 1. Mapa del estado de Querétaro y sus municipios.
Cuadro 1. Valores de las muestras para estimar ji-cuadrada, obteniendo los niveles de significancia para estandarizar la distribución de las muestras (ejemplares/área, ejemplares/visita y visitas/área).

\begin{tabular}{lrrr}
\hline Municipio & Área $\left(\mathrm{km}^{2}\right)$ & Visitas & Ejemplares \\
\hline Amealco & 674.81 & 4 & 4 \\
Arroyo Seco & 638.87 & 5 & 7 \\
Cadereyta & 1450.92 & 17 & 28 \\
Colón & 824.93 & 4 & 4 \\
Corregidora & 210.62 & 6 & 8 \\
El Marqués & 706.03 & 2 & 2 \\
Huimilpan & 362.61 & 3 & 3 \\
Jalpan & 933.36 & 1 & 2 \\
Landa de M. & 775.49 & 1 & 2 \\
Peñamiller & 657.69 & 6 & 10 \\
Pinal de A. & 605.88 & 4 & 6 \\
Querétaro & 734.07 & 5 & 5 \\
San Joaquín & 208.96 & 3 & 3 \\
San J. del Río & 798.98 & 1 & 2 \\
Tequisquiapan & 290.23 & 1 & 1 \\
Tolimán & 748.96 & 4 & 5 \\
Promedios & 666.49 & 4.19 & 5.75 \\
\hline
\end{tabular}

chnyanthes y Manfreda, con descripciones detalladas y comentarios taxonómicos para cada especie. El género Yucca fue estudiado ampliamente por Matuda y Piña (1980) quienes listan las especies distribuidas en México.

A nivel de regiones o estados, los estudios florísticos más sobresalientes son el de Gentry (1972), quien estudia la familia Agavaceae en Sonora, incluyendo los géneros Agave, Hesperaloë, Manfreda, Polianthes y Yucca. Tello y García (1988) estudian las especies del género Agave subg. Agave en el Altiplano PotosinoZacatecano, generando una lista florística y la distribución para esta zona, de las especies encontradas. McVaugh (1989) en Flora Novo-Galiciana incluye Agave, Furcraea, Manfreda, Polianthes, Prochnyanthes y Yucca. Synnott (1989) llevó a cabo un estudio florístico sobre los agaves de Nuevo León, donde menciona una pequeña descripción y comentarios acerca de las especies presentes. Galván (1990) describe las especies de los géneros Agave, Beschorneria, Furcraea, Manfreda, Polianthes y Yucca distribuidas en el Valle de México. Lott y García-Mendoza (1994) incluyen a los géneros Agave, Beschorneria, Furcraea, Manfreda, Polianthes y Yucca para la Flora Mesoaméricana.

Para el estado de Querétaro (figura 1), hasta antes de este trabajo no se habían hecho estudios profundos y específicos de las agaváceas. Sólo se contaba con datos bibliográficos aislados de algunos estudios florísticos. García-Mendoza y Galván (1995) mencio- 
nan las especies pertenecientes a esta familia en el estado. Argüelles et al. (1991) publican una lista florística del estado, en la que se mencionan algunas especies y su distribución en los municipios. Con respecto a los géneros, Zamudio y Sánchez (1995) describen una nueva especie de Agave de la Sierra Madre Oriental (incluyendo Querétaro). Howard (1985) registra una especie de Polianthes para el estado. Matuda y Piña (1980) describen las especies de Yucca y Piña (1989) describe una nueva especie de Yucca para Querétaro.

A nivel nacional sólo se cuenta con los estudios florísticos llevados a cabo por García-Mendoza (1995) y García-Mendoza y Galván (1995) quienes, como resultado de revisiones bibliográficas y de herbario, publican una lista de especies de Agavaceae en México y su distribución por estados. En dicho estudio se registra que Querétaro se encuentra en el lugar 23 de diversidad a nivel nacional.

Por esta razón se iniciaron estudios en la región para ampliar el conocimiento científico de este importante grupo de plantas. El objetivo propuesto fue el generar una lista de especies de Agavaceae en el estado de Querétaro y documentar sus áreas de distribución. Con esto se contribuye al conocimiento florístico del estado, iniciando las bases para llevar a cabo otro tipo de estudios (etnobotánicos, biogeográficos y ecológicos).

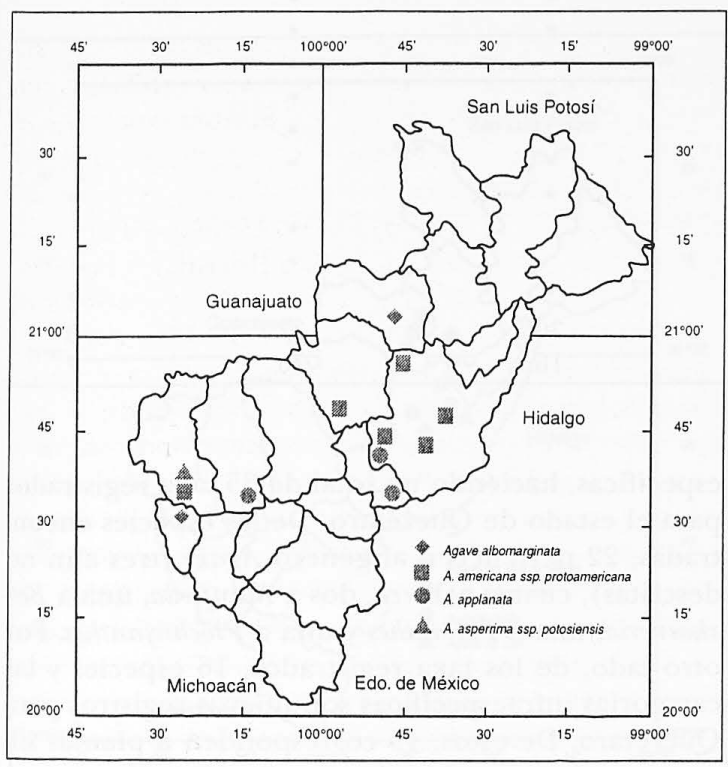

Figura 2. Distribución de Agave albomarginata, A. americana ssp. protamericana, $A$. applanata y $A$. asperrima ssp. potosiensis.

\section{Materiales y métodos}

Para la obtención de información, se revisaron ejemplares de la familia Agavaceae en los herbarios ENCB, IEB, MEXU y QMEX (siglas de acuerdo a Holmgren, 1990). Además de registrar datos morfológicos se anotó la información de las etiquetas de los ejemplares distribuidos en Querétaro.

Se hicieron 14 recorridos dentro del área de estudio, visitando 67 localidades para colectar los ejemplares de herbario y anotar las características ambientales y morfológicas. Con la ayuda del geoposicionador y mapas del estado de Querétaro se registró la localidad precisa de donde se colectaron las plantas (altitud, latitud y longitud) (cuadro 1). Los espacios se determinaron con las claves de Verhoek-Williams (1975), Matuda y Piña (1980), Gentry (1982) y McVaugh (1989). Para estimar el esfuerzo de colecta se llevaron a cabo análisis de ji-cuadrada utilizando el programa Statgraphics 6.0, obteniendo los niveles de significancia para estandarizar la distribución de las muestras (ejemplares/visita, ejemplares/área y visitas/ área). Finalmente, la información total se obtuvo a partir de un total de 195 ejemplares, 103 correspondiendo a revisiones de herbario y 92 a colectas llevadas a cabo en todo el estado de Querétaro, con un promedio de tres duplicados. La lista de especies y los mapas de distribución se ordenaron alfabéticamen-

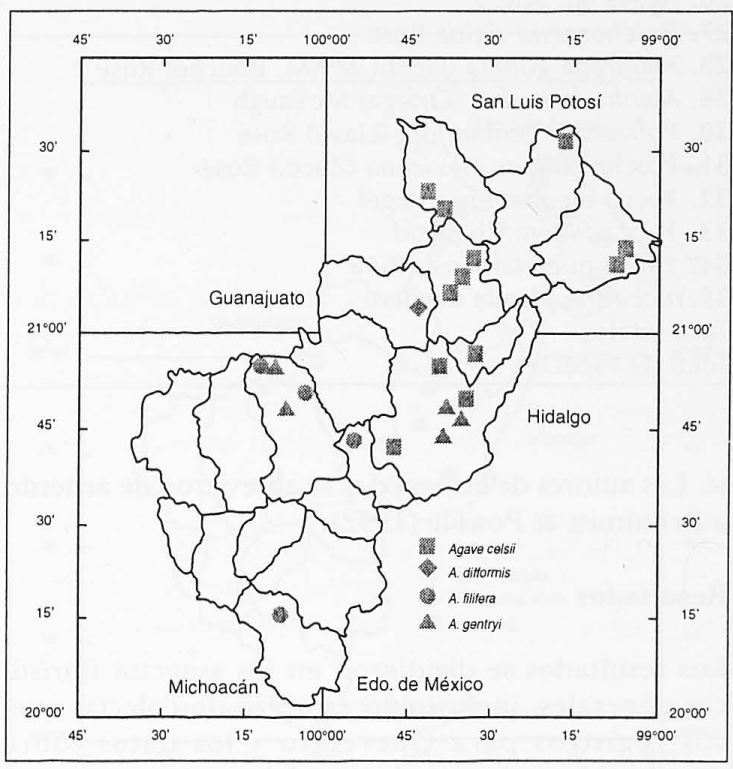

Figura 3. Distribución de Agave celsii, A. difformis, $A$. filifera y $A$. gentryi. 
Cuadro 2. Lista de especies de Agavaceae del estado de Querétaro, incluyendo nuevos registros, especies nativas e introducidas.

\begin{tabular}{|c|c|c|c|}
\hline Especies & $\begin{array}{l}\text { Nuevos } \\
\text { registros }\end{array}$ & $\begin{array}{l}\text { Plantas } \\
\text { nativas }\end{array}$ & $\begin{array}{l}\text { Plantas } \\
\text { cultivadas }\end{array}$ \\
\hline 1. Agave albomarginta Gentry & * & * & \\
\hline 2. Agave americana L. var. americana & & & * \\
\hline 3. Agave americana L. var. marginata Trel. & * & & * \\
\hline 4. Agave americana L. ssp. protamericana Gentry & * & $*$ & \\
\hline 5. Agave angustifolia Haw. var. rubescens (Salm-Dyck) Gentry & * & & * \\
\hline 6. Agave applanata K. Koch ex Jacobi & & * & \\
\hline 7. Agave asperrima Jacobi ssp. potosiensis (Gentry) Ullrich & & * & \\
\hline 8. Agave celsii Hook. & & * & \\
\hline 9. Agave desmettiana Jacobi & $*$ & & * \\
\hline 10. Agave difformis Berger & * & * & \\
\hline 11. Agave filifera Salm-Dyck & & * & \\
\hline 12. Agave gentryi Ullrich & * & * & \\
\hline 13. Agave aff. glomeruliflora (Engelm.) A. Berger & * & * & \\
\hline 14. Agave aff. heteracantha Zucc. & * & $*$ & \\
\hline 15. Agave lechuguilla Torr. & & $*$ & \\
\hline 16. Agave mapisaga Trel. var. Mapisaga & & & * \\
\hline 17. Agave sp. nov. Hernández et Magallán (inéd.) & * & * & \\
\hline 18. Agave salmiana Otto ex Salm-Dyck ssp. crassispina & * & * & \\
\hline 19. Agave salmiana Otto ex Salm-Dyck var. ferox & * & * & \\
\hline 20. Agave salmiana Otto ex Salm-Dyck var. salmiana & & & * \\
\hline 21. Agave schidigera Lem. & * & * & \\
\hline 22. Agave striata Zucc. ssp. striata & & * & \\
\hline 23. Agave tenuifolia Zamudio \& E. Sánchez & & * & \\
\hline 24.Agave xylonacantha Salm-Dyck & & * & \\
\hline 25. Agave sp. nov. 1 & * & * & \\
\hline 26. Agave sp. nov. 2 & * & * & \\
\hline 27. Beschorneria rigida Rose & * & * & \\
\hline 28. Manfreda guttata (Jacobi \& P.M. Bouché) Rose & & * & \\
\hline 29. Manfreda scabra (Ortega) McVaugh & * & * & \\
\hline 30. Polianthes geminiflora (Llave) Rose & & * & \\
\hline 31. Prochnyanthes mexicana (Zucc.) Rose & * & $*$ & \\
\hline 32. Yucca elephantipes Regel & * & & $*$ \\
\hline 33. Yucca filifera Chabaud & & * & \\
\hline 34. Yucca queretaroensis Piña & & * & * \\
\hline 35. Yucca treculeana Carrière & * & & $*$ \\
\hline Taxa totales & 20 & 27 & 8 \\
\hline Total de especies & 16 & 26 & 7 \\
\hline
\end{tabular}

te. Los autores de las especies se abreviaron de acuerdo a Brummitt \& Powell (1992).

\section{Resultados}

Los resultados se dividieron en los aspectos florísticos generales, incluyendo esfuerzo de colecta, nuevos registros para Querétaro y los datos sobre distribución y riqueza dentro del estado.

Se encontraron seis géneros de Agavaceae (Agaze, Beschorneria, Manfreda, Polianthes, Prochnyanthes y Yucca) con 31 especies, además de cuatro categorías infra- específicas, haciendo un total de 35 taxa registrados para el estado de Querétaro. De las especies encontradas, 22 pertenecen al género Agave (tres aún no descritas), cuatro a Yucca, dos a Manfreda, una a Beschorneria, una a Polianthes y una a Prochnyanthes. Por otro lado, de los taxa registrados, 16 especies y las categorías infraespecíficas son nuevos registros para Querétaro. De estos, 15 corresponden a plantas silvestres y cinco a cultivadas (cuadro 2).

Se registra por primera vez la presencia de los géneros Beschorneria y Prochnyanthes en el estado, así como la distribución natural de Agave albomarginata. 
Beschorneria rigida conocida de Guanajuato, San Luis Potosí y Tamaulipas, se localizó en un bosque mesófilo del municipio de Pinal de Amoles. Prochnyanthes mexicana se registró al suroeste del estado, en la provincia fisiográfica del Eje Volcánico Transversal, en un bosque de Quercus del municipio de Huimilpan. Su distribución se conocía desde Durango y Nayarit hasta Michoacán. Finalmente, la especie Agave albomarginata descrita por Gentry (1982) a partir de ejemplares del Jardín Botánico de Huntington, California, se registró por primera vez en el centro y sur del estado. Debe mencionarse que la especie designada como Agave aff. heteracantha al colectarse por primera vez se pensó que era una especie no descrita, sin embargo, al revisar los trabajos de Berger (1915) se encontró una descripción que concuerda en gran parte con las características de esta planta. Para clarificar la identidad y nomenclatura de esta especie se requiere de mayor investigación documental y revisión de los ejemplares tipo.

Las especies con la distribución más amplia en Querétaro son Yucca filifera, que se encuentra en 16 municipios, siendo más abundante hacia la porción central del estado y Agave celsii, que se colectó en seis municipios, restringido a la provincia de la Sierra Madre Oriental es particularmente abundante en los municipios de Pinal de Amoles y San Joaquín. Las especies cultivadas como A. americana, A. mapisaga y
A. salmiana también se encuentran ampliamente distribuidas en la entidad por su uso generalizado. Esto no es muy notable en los mapas presentados, pues en muchas ocasiones no fueron colectados por no presentar inflorescencia, sin la cual era muy difícil saber la variedad o subespecie. Sin embargo, se observaron en la mayor parte del estado, generalmente en estrecha relación con actividades humanas. La distribución de todas las especies se observa en las figuras 2 a 8 .

El esfuerzo de colecta es rara vez estimado en los trabajos florísticos por lo que no existen datos comparativos con otras entidades o regiones. En el caso particular se hicieron pruebas de independencia (jicuadrada), estimando los valores de significancia para las relaciones esperadas entre número de ejemplares por visita, número de visitas por tamaño de cada municipio y número de ejemplares por área de cada municipio con las observadas. En todos los casos se consideraron 15 grados de libertad. Para la relación entre el número de ejemplares por visita no se encontraron diferencias significativas $(P=0.9955468)$ entre los dos modelos, lo que sugiere que no se sobremuestreó o submuestreó en ninguna de las localidades. Con respecto al número de visitas y al número de ejemplares colectados por área de cada municipio, se encontraron diferencias significativas en los dos casos $(\mathrm{P}=0.0024059$ en el primero y $\mathrm{P}=0.00000141$

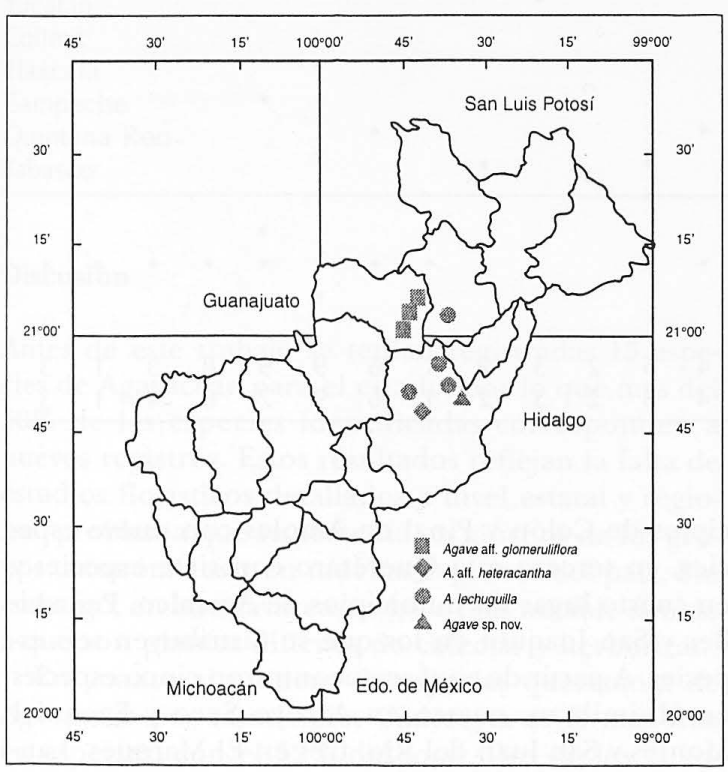

Figura 4. Distribución de Agave aff. glomeruliflora, $A$. aff. heteracantha, A. lechuguilla y Agave sp. nov.

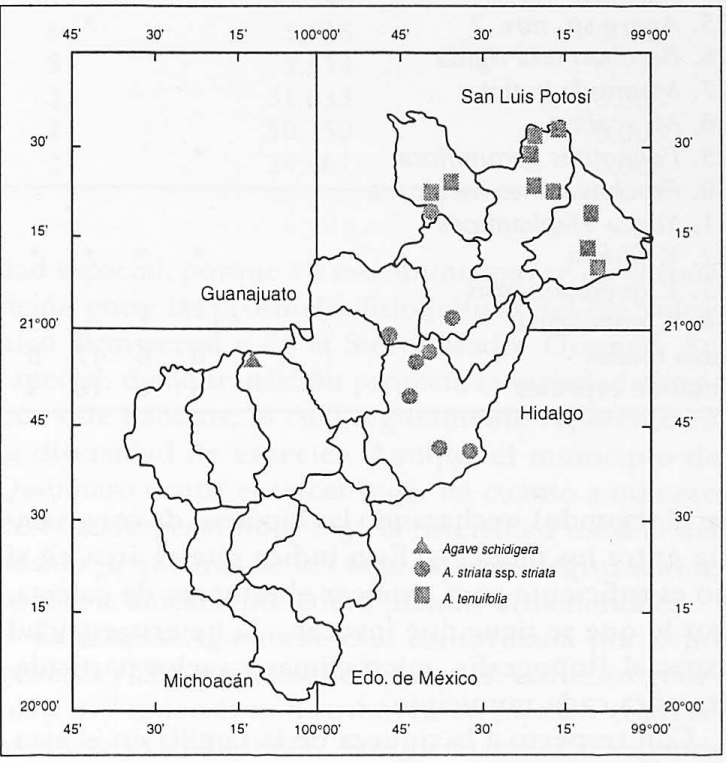

Figura 5. Distribución de Agave schidigera, A. striata ssp. striata y $A$. tenuifolia. 
Cuadro 3. Especies registradas en cada municipio del estado de Querétaro. Claves de cada municipio (Argüelles et al., 1991). $\mathrm{A}=\mathrm{Amealco}, \mathrm{AS}=$ Arroyo seco, $\mathrm{C}=$ Cadereyta, $\mathrm{CO}=$ Colón, $\mathrm{CR}=$ Corregidora, $\mathrm{EM}=\mathrm{Eze}-$ quiel Montes, $\mathrm{H}=$ Huimilpan, J= Jalpan, $\mathrm{LM}=$ Landa de Matamoros, $M A=E I$ Marqués, $P E=P e d r o$ Escobedo, $\mathrm{PM}=$ Peñamiller, $\mathrm{PA}=$ Pinal de Amoles, $\mathrm{Q}=$ Querétaro, $\mathrm{S} J=$ San Joaquín, $\mathrm{SR}=\mathrm{San}$ Juan del Río, $\mathrm{T}=$ Tequisquiapan y $\mathrm{TO}=$ Toliman.

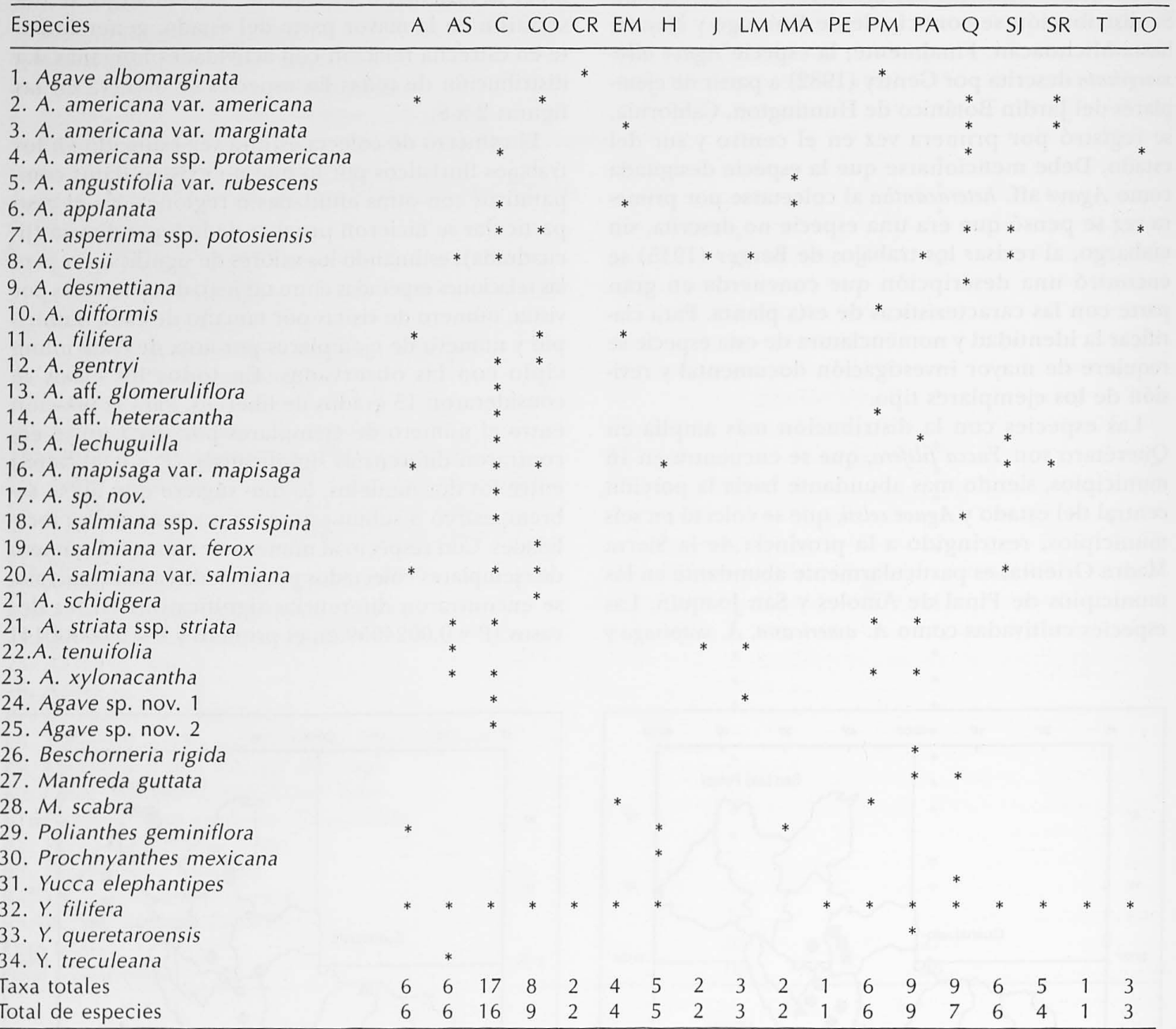

en el segundo), rechazando las hipótesis de congruencia entre los modelos. Esto indica que el área en sí no es suficiente para explicar el esfuerzo de colecta, por lo que se tiene que invocar a la heterogeneidad espacial (topografía, microclimas y suelos particulares para cada municipio).

Con respecto a la riqueza de la familia en el estado, el municipio con mayor número de especies es Cadereyta, ocurriendo $16(52 \%)$ de las 31 especies encontradas. En segundo lugar tenemos a los muni- cipios de Colón y Pinal de Amoles con nueve especies, en tercero está Querétaro con siete especies y en cuarto lugar los municipios de Amealco, Peñamiller y San Joaquín en los que se distribuyen seis especies. A partir de aquí se encontraron cinco especies en Huimilpan, cuatro en Arroyo Seco y Ezequiel Montes y San Juan del Río, tres en El Marqués, Landa de Matamoros y Tolimán, dos en Jalpan y Corregidora y una en Pedro Escobedo y Tequisquiapan (cuadro 3). 
Cuadro 4. Distribución de Agavaceae por estados de acuerdo a la relación especies área (Modificado de GarcíaMendoza y Galván, 1995).

\begin{tabular}{|c|c|c|c|}
\hline Estado & $\begin{array}{c}\text { Total } \\
\text { de spp. }\end{array}$ & $\begin{array}{c}\text { Áreas } \\
\text { en } k m 2\end{array}$ & $\begin{array}{c}\text { Especies } \\
\text { /área }\end{array}$ \\
\hline Zacatecas & 29 & 5,364 & 0.540 \\
\hline Morelos & 16 & 4,941 & 0.323 \\
\hline Querétaro & 31 & 11,769 & 0.269 \\
\hline México & 29 & 21,461 & 0.135 \\
\hline Hidalgo & 27 & 20,897 & 0.129 \\
\hline Puebla & 43 & 33,919 & 0.126 \\
\hline Oaxaca & 52 & 95,364 & 0.054 \\
\hline San Luis Potosí & 33 & 64,848 & 0.050 \\
\hline Jalisco & 40 & 80,187 & 0.049 \\
\hline Nuevo León & 29 & 64,555 & 0.045 \\
\hline Michoacán & 24 & 59,864 & 0.040 \\
\hline Guerrero & 23 & 63,794 & 0.036 \\
\hline Veracruz & 25 & 72,815 & 0.034 \\
\hline Tamaulipas & 26 & 79,829 & 0.032 \\
\hline Chiapas & 24 & 73,887 & 0.032 \\
\hline Coahuila & 35 & 151,571 & 0.023 \\
\hline Sonora & 40 & 184,934 & 0.021 \\
\hline Baja California & 13 & 70,113 & 0.018 \\
\hline Chihuahua & 34 & 247,087 & 0.013 \\
\hline Durango & 43 & 111,648 & 0.003 \\
\hline Nayarit & 22 & 27,621 & 0.079 \\
\hline Baja California Sur & 19 & 73,677 & 0.025 \\
\hline Guanajuato & 19 & 30,589 & 0.062 \\
\hline Sinaloa & 18 & 58,092 & 0.030 \\
\hline Aguascalientes & 12 & 5,589 & 0.214 \\
\hline Distrito Federal & 11 & 1,499 & 0.733 \\
\hline Yucatán & 7 & 39,340 & 0.017 \\
\hline Colima & 6 & 5,455 & 0.109 \\
\hline Tlaxcala & 5 & 3,914 & 0.127 \\
\hline Campeche & 3 & 51,833 & 0.005 \\
\hline Quintana Roo & 2 & 50,350 & 0.003 \\
\hline Tabasco & 2 & 24,661 & 0.008 \\
\hline
\end{tabular}

\section{Discusión}

Antes de este trabajo se tenían registradas 15 especies de Agavaceae para el estado, por lo que más del $50 \%$ de las especies identificadas corresponden a nuevos registros. Estos resultados reflejan la falta de estudios florísticos detallados a nivel estatal y regional, problema que se presenta en muchos de los grupos taxonómicos y en muchas regiones del país. Sin embargo, en las agaváceas se hace más notable debido a que son plantas difíciles de colectar y herborizar.

En cuanto al número de especies queretanas de Agavaceae por municipio, Cadereyta es el más rico en especies, con el $52 \%$ del total. Dos de los factores que seguramente están influyendo en la alta diversidad del municipio son, su extensión territorial, que es de las mayores en el estado y su heterogenei- dad espacial, porque en este municipio se da la transición entre las provincias fisiográficas del Eje Volcánico Transversal y de la Sierra Madre Oriental. En especial, dicha transición propicia la variedad climática y de hábitats, lo cual seguramente repercute en la diversidad de especies. Aunque el municipio de Querétaro ocupa el tercer lugar en cuanto a número de especies, esto no refleja la diversidad natural del mismo ya que tres de sus siete especies registradas se conocen únicamente como plantas ornamentales.

La familia Agavaceae está conformada por ocho géneros y 288 especies, de los cuales, todos los géneros y 217 especies se distribuyen en México (GarcíaMendoza y Galván, 1995). En Querétaro las 31 especies resultantes (incluyendo silvestres y cultivadas) corresponden al $14.3 \%$ del total para México. Si se excluyen las cuatro especies no silvestres o que únicamente 
se encontraron de manera cultivada, ya sea con fines ornamentales o de otros usos (Agave desmettiana, A. mapisaga, Yucca elephantipes y Y. treculeana) las 27 restantes corresponden entonces al $13 \%$ del total para el país. En este punto es importante destacar que no se excluyen especies como Agave americana y A. salmiana, ya que ambas especies tienen categorías infraespecíficas en forma silvestre (Agave americana ssp. protamericana y A. salmiana ssp. crassispina).

De acuerdo con García-Mendoza y Galván (1995) Querétaro es menos diverso que los estados de Oaxaca, Durango, Puebla, Jalisco, Sonora, Coahuila, Chihuahua y San Luis Potosí, que tienen un territorio mucho mayor. Sin embargo, si se compara el número de especies por estado considerando su extensión territorial, se observa que Querétaro es el tercer estado más diverso en especies de Agavaceae después de Zacatecas y Morelos (cuadro 4).

En los estudios llevados a cabo por García-Mendoza (1995) se menciona que el Valle de Tehuacán-Cuicatlán se encuentra entre las zonas más diversas del país por presentar 20 especies en un cuadro de un grado por lado. Al comparar estos resultados con los del presente trabajo, en un cuadro de un grado por lado $\left(99^{\circ} 15^{\prime}-100^{\circ} 15^{\prime}\right.$ long. $\mathrm{N}$ y $20^{\circ} 30^{\prime}-21^{\circ} 30^{\prime}$ lat. W), correspondiente a la zona y que incluye los municipios o porciones de Arroyo Seco, Cadereyta, Colón, Ezequiel Montes, Jalpan, Landa de Matamoros, Pe- ñamiller, Pinal de Amoles, San Joaquín, Tequisquiapan y Tolimán, se encontraron 24 especies silvestres de Agavaceae. Por lo que esta zona conocida regionalmente como Semiárida Queretana en la vertiente occidental de la Sierra Madre Oriental o Sierra Gorda de Querétaro, presenta mayor diversidad que la observada para el Valle de Tehuacán-Cuicatlán. Es obvio que el número de especies registrado por estado no sólo se debe a la diversidad real de cada uno, sino también a la intensidad de muestreos y trabajos florísticos detallados. Por esta razón nos parece valido comparar Querétaro con el Valle de TehuacánCuicatlán, ya que es una región en la cual se han llevado a cabo estudios florísticos intensivos y por lo tanto un número considerable de muestreos. Dada la gran diversidad de México, y la falta de estudios específicos, en cualquier grupo taxonómico o región del país donde se lleven a cabo muestreos intensivos, invariablemente el número de especies aumentará con respecto a los registros anteriores.

Con respecto al resto del país, es muy probable que la diversidad de Querétaro, a pesar de tener una extensión territorial relativamente reducida, se deba a que en él confluyen tres grandes regiones fisiográficas que repercuten directamente en la heterogeneidad de climas y hábitats. Igualmente, la familia Agavaceae presenta géneros tanto con afinidad a zonas templadas y subhúmedas (Beschorneria, Manfreda,

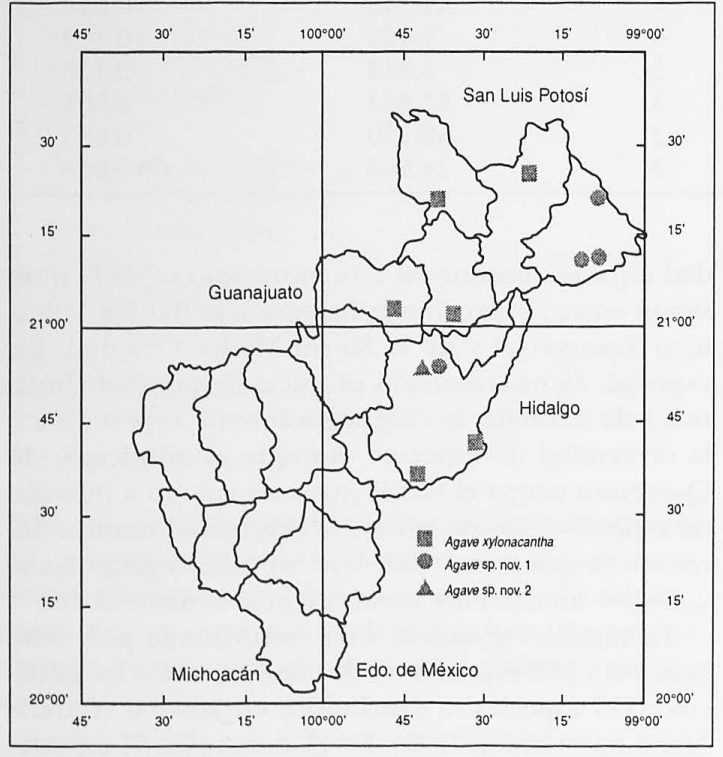

Figura 6. Distribución de Agave xylonacantha, Agave sp. nov. 1 y Agave sp nov. 2.

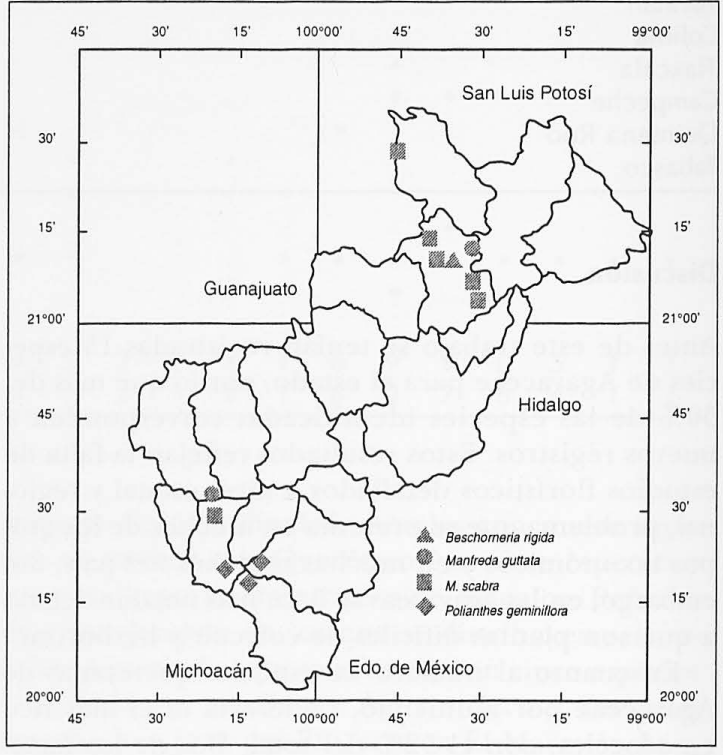

Figura 7. Distribución de Beschorneria rigida, Manfreda guttata, M. scabra y Polianthes geminiflora. 
Polianthes y Prochnyanthes), como con afinidad a zonas cálidas y secas (Agave y Yucca).

Finalmente, se resalta la importancia, en términos florísticos, culturales y económicos, de las plantas de esta familia a nivel regional, sobre todo para los campesinos y grupos indígenas. Es necesario comentar la importancia que tienen los estudios florísticos, no sólo por el hecho de que gracias a éstos se puede conocer la distribución del grupo de interés, sino también porque representan la base para cualquier trabajo etnobotánico, biogeográfico, ecológico o de conservación. En adición, el hecho de conocer las especies que se encuentran en el estado, su distribución y su hábitat, puede repercutir directamente en beneficio de los queretanos. Muchas de las especies, como Agave albomarginata, A. celsii, A. filifera, A. aff. heteracantha, A. sp. nov., A. schidigera, A. xylonacantha, Prochnyanthes mexicana y Yucca queretaroensis pueden ser cultivadas con fines ornamentales, tanto por su gran belleza como por tratarse de plantas poco comunes.

\section{Agradecimientos}

Queremos expresar nuestro agradecimiento a Abisaí García-Mendoza, Luis Eguiarte y Raquel Galván por su apoyo en la publicación del artículo, por sus revisiones y acertadas sugerencias. A Mahinda Martínez y Maricela Gómez por la revisión crítica del manus-

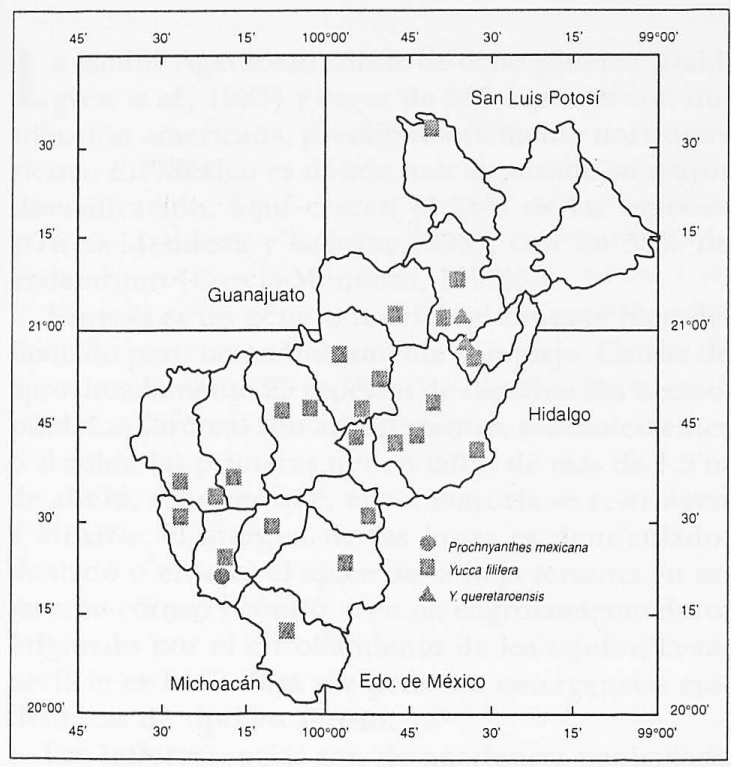

Figura 8. Distribución de Prochnyanthes mexicana, Yucca filifera y $Y$. queretaroensis. crito. A los curadores de los herbarios ENCB, IEB, MEXU y QMEX por facilitarnos la consulta de material. A Jacinto Treviño y a Olivia Baltazar por su ayuda en las salidas de campo. A Javier Ponce por su ayuda en los análisis estadísticos.

\section{Literatura citada}

Argüelles E., Zamudio S. y Fernández R. 1991. Flora del Bajio $y$ de regiones adyacentes. Listado florístico preliminar del estado de Querétaro. Instituto de Ecología A.C. Centro regional del Bajío, Pátzcuaro Michoacán y CONCYTEQ. Fascículo complementario II.

Berger A. 1915. Die Agaven. Beitrage zu einer monographie. Verlag von Gustav Fisher Jena.

Brummit R.K. y Powell C.I. 1992. Authors of plant names. Royal Botanic Gardens Kew.

Dahlgren R.M.T., Clifford H.T. y Yeo P.F. 1985. The families of the monocotyledons. Structure, evolution and taxonomy. Springer Verlag. Berlín.

Galván R. 1990. Liliaceae, Amaryllidaceae. En: Rzedowski J y Rzedowski G.C. Eds. Flora Fanerogámica del Valle de México. Instituto de Ecología A.C. Centro regional del Bajío. Pátzcuaro, Michoacán. Vol. III: 289-320.

García-Mendoza A. 1987. Monografía del género Beschorneria Kunth. Agavaceae. Tesis maestría, UNAM, México, $131 \mathrm{pp}$.

García-Mendoza A. y Galván-Villanueva R. 1995. Riqueza de las familias Agavaceae y Nolinaceae en México. Boletín de la Sociedad Botánica de México. 56:7-24.

García-Mendoza A. 1995. Riqueza y endemismo de la familia Agavaceae en México. En: Linares E., Dávila P., Chiang F., Bye R. y Elias T. Eds. Conservación de plantas en peligro de extinción. Diferentes enfoques. UNAM, México, $51-75$.

Gentry H.S. 1972. The Agave Family in Sonora. Agricultural Research Service U.S. Department of agriculture. Agriculture Handbook 399.

Gentry H.S. 1982. Agaves of continental North America. The University of Arizona Press. Tucson.

Gonçalves D.L.O. 1978. El maguey y el pulque en los códices mexicanos. Fondo de Cultura Económica. México.

Granados S.D. 1993. Los Agaves de México. Universidad Autónoma de Chapingo. México.

Hernández L. 1998. Sistemática de Agavaceae y Nolinaceae: una perspectiva descle el siglo VIII. Biología Informa, Boletin informativo de la Licenciatura en Biologia. UAQ. 9:6-11.

Holmgren P.K., Holmgren N.H. y Barnett L. 1990. Index Herbariorum Part I. The Herbaria of the World. $8^{\text {th }}$ edition. New York Botanical Garden. New York.

Howard T.M. 1985. Stalking the Polianthes of México. Herbertia. 42:98-117.

Matuda E. y Piña L.I. 1980. Las plantas mexicanas del género Yucca. Gobierno del estado de México, México. 
McVaugh R. 1989. Liliaceae. En: Anderson W.R. Ed. Flora Novo-Galiciana. A descriptive account of the vascular plants of western Mexico. Ann Arbor; The University of Michigan Herbarium. Vol. 15:120-293.

Lott J.E. y García-Mendoza A. 1994. Agavaceae. En: Davidse G. et al. Flora Mesoamericana. Volumen 6. Universidad Nacional Autónoma de México, Instituto de Biología, México D.F., 35-47.

Ojeda-Revah L. y Ludlow Wiechers B. 1995. Palinología de Agavaceae, una contribución biosistemática. Boletín de la Sociedad Botánica de México. 56:25-43.

Piña L.I. 1989. Una nueva especie de Yucca (Agavaceae). Cactáceas y Suculentas Mexicanas. 34(3):51-56.

Rose J.N. 1903-1905. Amaryllidaceae. Revision of Polianthes whit new species. Prochnyanthes and its species. Manfre$d a$ and its species. Contributions from The United States National Herbarium 1:8-23.
Rzedowski J. 1978. La vegetación de México. Limusa. México. Synnott T.J. 1989. Informe sobre los Agaves de Nuevo León. Cactáceas y Suculentas Mexicanas. 34:64-72.

Tello J.J. y García E. 1988. El maguey (Agave, subgénero Agave) en el altiplano potosino-zacatecano. Boletín de la Sociedad Botánica de México. 48:119-134.

Trelease W. 1920. Amaryllidaceae. En: Standley P.C. Ed. Trees and Shrubs of Mexico. Contributions from the United States National Herbarium. 23:107-142.

Verhoek-Williams S. 1975. A study of the tribe Poliantheae (including Manfreda) and revisions of Manfreda and Prochnyanthes (Agavaceae). Ph. D. Thesis. Cornell University. U.S.A., 405 pp.

Zamudio S. y Sánchez E. 1995. Una nueva especie de Agave del subgénero Littaea (Agavaceae) de la Sierra Madre Oriental, México. Acta Botánica de Mexicana. 32:47-52. 